\title{
A Framework for Heterogeneous Home Monitoring WSN Networks
}

\author{
Ch. P. Antonopoulos ${ }^{* 1}$, G. Touliatos ${ }^{1}$, Ch. Panagiotou ${ }^{1,2}$, Th. Panagiotakopoulos ${ }^{2}$, \\ S. Koubias ${ }^{1}$, Achilles Kameas ${ }^{2}$, Nikolaos S. Voros ${ }^{3}$ \\ 1Applied Electronics Laboratory, ECE Dept, University of Patras, Greece \\ 2 e-CoMeT Lab, Hellenic Open University, Patras, Greece \\ ${ }^{3}$ Computer and Informatics Engineering Department, Technological Educational Institute of Western Greece \\ ${ }^{(*)}$ Corresponding author email: cantonop@ece.upatras.gr
}

\begin{abstract}
During the last years Home Monitoring has emerged as a prominent research and development area able to offer significant services to a wide variety of application scenarios. Without a doubt, factors of critical importance that propelled respective interest include advancements in ICT areas such as wireless sensor networks (WSNs), hardware design and adequate sensors' circuitry implementation. However, most of the existing solutions focus on the use of specific and even custom technologies which result into systems of limited usefulness, flexibility and extensibility unable to cover all aspects of home monitoring. Aiming to address such inefficiencies this paper presents the design and implementation of the ADVENT Home Monitoring system embracing heterogeneity in various aspects. At the same time it is of paramount importance for the system to be well accepted by all users regardless to their technological or educational background. Therefore, considerable effort has been devoted so as to present the underlying heterogeneous system in a homogenous, user friendly and attractive way to the end user. Furthermore, a critical goal is to present a continuously extending system able to adopt new ICT advancement and thus be versatile in order to cover different and diverse user required services and applications.
\end{abstract}

Keyword: Ambient Assisted Living, Home Monitoring, Wireless Sensor Networks, Bluetooth, IEEE 802.15.4.

\section{INTRODUCTION}

Based on projections concerning various aspects of everyday life characteristics it is argued that efficient and comprehensive home monitoring will comprise a critical necessity in the near future. Particularly, taking advantage of advancements in medical fields the life expectancy is constantly increasing and it is expected to keep on increasing in the near future [1]. Consequently, the number of elderly people in need of constant care and attention will increase accordingly. At the same time covering such needs through existing approaches and services such as hospitalization, nursing homes or employing personal care givers at home for a continuously increasing population percentage is not a viable solution. This is due to practical [2], economical [3] and psychological [4] reasons.

From another perspective it must also be emphasized that home monitoring (not only for elderly people but in general) is a highly personalized and multifaceted challenge. First of all the reasons a person may need or want home monitoring can be quite diverse. On one hand a typical category are the users suffering from specific chronic conditions. Even more, the actual condition (e.g. kinetic difficulties, heart conditions, brain conditions etc.) and the needs for each specific user are diverse (or diversified)and thus a "one size fits all" solution is not adequate. On the other hand, an elderly person may not have any specific condition or/and disease, but home monitoring is needed because he/she spends a lot of time on its own and a general observation on the user's wellbeing is desired. Of course home monitoring focusing more on safety issues (e.g. the user forgot to turnoff the oven, or close a window before going to sleep etc.) also comprise a service of high added value for the general population [5].

In face of these challenges ICT advancements in many areas such as Wireless Sensor Networks, hardware design and miniaturization, pose a prominent solution [6-8]. Indeed over the last years increasing number of WSN platforms have appeared offering various monitoring capabilities. However, most commercial solutions tend to lack the required degree of flexibility so as to offer truly personalized and adaptive services. Furthermore, commercial as well as research solutions tend to focus on specific areas of monitoring and use specific technologies and sensors, thus omitting to cover other equally important needs [9-10]. Consequently, we end up with a wide variety of, in various aspects, heterogeneous systems including: WSN communication technologies, sensors able to acquire physiological, kinetic and ambient data, hardware characterized by different processing, storage and energy capabilities [11], [12]. Aiming to enhance the utilization and usefulness of WSN technologies in home monitoring the main objective of this paper is to present critical design and implementation aspects of a platform able to take advantage of different underlying communication technologies, available sensors and hardware. In this way, the goal of such platform is to offer the user a truly comprehensive and highly personalized home monitoring environment as envisioned in the ADVENT research project [19].

The rest of this paper is structured as follows. Section II aims to present the design and implementation characteristics of the aggregation point of the ADVENT home environment. Section III focuses on the WSN section of home environment. Finally Section IV discusses the main conclusions and presents the future directions of this ongoing effort.

\section{HOME GATEWAY FOR HETEROGENEOUS WSNS}


Acting as the controller and aggregation point of the sensor data, the Home Gateway (identified from now on as $\mathrm{HG}$ ) is of cornerstone importance in the context of the ADVENT Home Environment. A dominant part in recent ICT trends is covered by an evolution in wearable devices and sensors in a context of ubiquitous environment and Ambient Assisted Living (AAL) solutions. Multiple sensing devices can be found in the market from respectively various vendors introducing the challenge of interoperability and cooperation between them. These issues comprised the main motivation leading to the design principles of the ADVENT HG which can be described by the following main functionalities:

1. Support for/of multiple network interfaces

2. Support for/of multiple types of sensors

3. Data aggregation and temporary storage capabilities

4. Data processing

5. User interface

Considering all the above, the HG functionality can be divided into three main blocks as depicted in Fig.1.

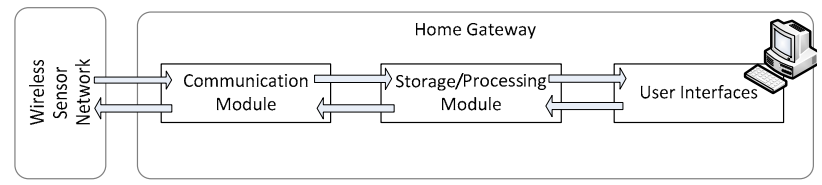

Figure 1. Data Flow Path in ADVENT Home Environment

\section{A. Communication Module}

As depicted in Fig. 1, the Communication Module represents the entry point of data to the HG. This module is responsible for the interconnection with the medical/monitoring devices and faces the main challenge of handling multiple communication processes with heterogeneous devices and gathering the generated data into a single system. For these purposes, we adopted open source technologies and more specifically the GNU/Linux [16] as the HG's operating system while our software is developed in Python [17] as a widely used, flexible, general purpose, high level programming language.

At the moment two major standardized communication technologies are supported, the IEEE 802.15.1 (Bluetooth) [15] and IEEE 802.15.4 [14]. The main design and implementation challenge was to integrate the communication drivers for each device and offer them to the upper application layers as a "black box". The communication module exposes an interface to the rest of the application with a higher layer of abstraction hiding the communication details in terms of communicating protocols and technology.

Before describing the communication process among the wireless devices and the ADVENT HG, first the packets' structure that are exchanged among the HG and the wireless sensors must be presented. The basis of the application communication protocol was the open source ShimmerConnect application provided by Shimmer [11], which we further modified and extended with new capabilities. The three main categories of commands existing in ShimmerConnect and retained in the extended version are as follows:

A. SET: The set commands are used for sensor configuration (enabling/disabling sensors, set sampling rate, calibration parameters, etc)

B. GET: The get commands are used for sensing devices' configuration (sampling rates, sensor ranges, etc). INQUIRY COMMAND get command is probably the most important get command triggering the transmission of INQUIRY_RESPONSE containing the entire configuration of the unit.

C. ACTION: Finally the action commands are responsible for starting and stopping the data streaming

After every single command the wireless node receives, it replies with a respective acknowledgement message back to the HG. If an additional message is required to be sent, then the acknowledgement indication is merged with this message in one packet.

So far we have highlighted the high level features of the HG capability to support multiple sensors and vendors. In that context, a critical enhancement was the extension of the "sensing device's profile" concept. This profile consists of a node type id that is associated with a respective vendor and stored in the HG in a database table. The profile is further populated by the parameters that the sensor provides through the GET commands. Since the node type id is reported by the WSN node a respective field was added in the message structure of the information inquiry GET command. Extracted from respective GET command, information is acquired describing the respective Shimmer device but it is further required to distinguish multiple devices having the same configuration, e.g. two ShimmerSensing accelerometer sensor boards. Therefore an additional field was added in the structure of every packet sent by the Shimmer Boilerplate denoting the unique id for each connected device. An example of a typical INQUIRY_RESPONSE packet as formulated in the ADVENT Home Environment context is depicted in Table 1 (the two new fields are shaded in grey).

TABLE 1: ADVENT INQUIRY RESPONSE PACKET

\begin{tabular}{|c|c|c|c|c|c|c}
\hline Byte & 0 & 1 & 2 & 3 & 4 & $\ldots$ \\
\hline Value & $\begin{array}{c}\text { Packet } \\
\text { Type }\end{array}$ & $\begin{array}{c}\text { Node } \\
\text { Unique } \\
\text { Id }\end{array}$ & $\begin{array}{c}\text { Node } \\
\text { Type } \\
\text { Id }\end{array}$ & $\begin{array}{c}\text { Sampling } \\
\text { rate }\end{array}$ & $\begin{array}{c}\text { Accel } \\
\text { Range }\end{array}$ & $\ldots$ \\
\hline
\end{tabular}

\begin{tabular}{c|c|c|c|c|c|c|c|}
\hline$\ldots$ & 5 & 6 & 7 & 8 & 9 & $\ldots$ & $\mathrm{X}$ \\
\hline$\ldots$ & $\begin{array}{c}\text { Config } \\
\text { Byte 0 }\end{array}$ & $\begin{array}{c}\text { Number of } \\
\text { Channels }\end{array}$ & $\begin{array}{c}\text { Buffer } \\
\text { Size }\end{array}$ & Chan1 & Chan2 & $\ldots$ & ChanX \\
\hline
\end{tabular}

\section{B. Communication Process}

Initially, a background thread is responsible for listening for devices following the Serial Port Profile (SPP). The interface between the wireless devices and the HG is based on serial communication protocols through respective ports e.g. RFCOMM $x$ or ttyUSB $x$. Additionally, for the Bluetooth module implementation the PyBluez [18] python library was utilized, which is a collection of wrappers around the system's Bluetooth resources.

The application layer communication begins provided that the underlying respective connection is established. Sequentially the HG sends an INQUIRY_COMMAND and waits for the INQUIRY_RESPONSE of the wireless device that will announce to the HG its network wide unique ID and node type ID. The HG searches for the specific node type ID to the respective table in order to identify the 
Vendor and the Sensor set that this node type ID is associated to. Following the completion of the initialization step of the communication, the HG sends the respective start streaming action command to the sensing device. It is noted that the HG is capable to determine dynamically the length and the structure of the packet through the device profiles. The data packet structure and the streaming data are then passed to another fundamental module of the HG the data packet parser. The data packet parser is a Python software module that is responsible to extract useful information from the data packets and store it to a SQLlite based database.

\section{WSN NODE SOFTWARE ARCHITECTURE}

In this section we highlight the main design and implementation issues addressed focusing on the WSN part of the home environment

\section{A. ADVENT Mote Firmware Application Layer}

Our software implementation is based on Shimmer's BoilerPlate firmware [12] which is adequately enhanced to support different communication technologies, platforms and sensors. According to the ADVENT vision, each mote can be equipped with a variety of sensors from different vendors, such as accelerometer, ECG, Temperature /Pressure, etc. Its main functionality is to sample available sensors, wrap their values in data packets and transmit them via IEEE 802.15.4 or Bluetooth wireless connections. Moreover, it supports a variety of commands, so that the user can be aware of the available sensors to each mote, the parameters' values, such as the sampling rate, and also able to reconfigure them. The general architecture of this application is presented in Fig. 2 .

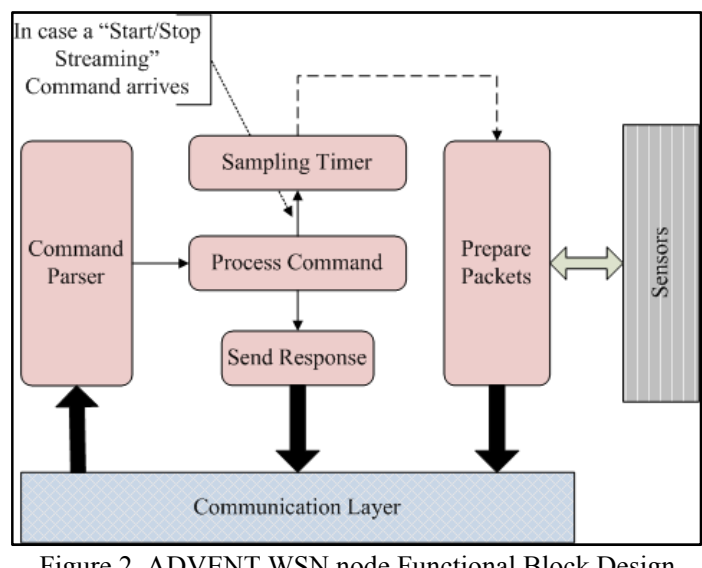

Figure 2. ADVENT WSN node Functional Block Design

Accordingly, the functionality of this application can be divided in three main operations. The first of them is initialization. During this phase, all required initial values are set, including a predefined set of configurations, such as the sampling rate and the pre-selected set of sensors with respect to the vendor. The second main operation is the one responsible for receiving and responding to commands. Specifically, the Communication Module sends every byte it receives to the Command Parser module effectively hiding the specific communication protocol. The latter assembles incoming bytes to commands forwarded to the Process Command functional module. This function, for every command, performs the appropriate operations as depicted in Fig. 2. Specifically, if a configuration command arrives, then it applies the required configuration changes. However, in case a response is required, it sets the appropriate flags and the control proceeds to the depicted Send Response functional module. The latter assembles the required response in a data packet, which is conveyed to the communication module in order to be sent. Finally, the third branch of actions begins when a "start streaming command" arrives. This command, instructs the mote to start transmitting data from the sensors.

Respective support required significant enhancements and modifications at the functional module responsible for the assembly and sending of the response to the communication module. Moreover, and in accordance to the ADVENT HG design, modifications where required concerning the format of the data packets so as to be compatible with the demands of our application as presented in the previous section. Finally, fine tuning modifications were made regarding the internal transmission process in order to support transparent packet transmission regardless of the considered communication protocol.

\section{B. ADVENT Mote Communication Module Implementation}

As stated in the introduction, the presented framework aims to be flexible and extensible concerning the support of prominent communication techniques and specifically IEEE 802.15.4 and Bluetooth. To achieve this goal, significant effort was devoted to IEEE 802.15.4 support, since Shimmer firmware offers only Bluetooth communication.

Two are the major differences between Bluetooth and 802.15.4 in the context of our implementation. First, communication via Bluetooth is byte oriented, while 802.15.4 relies on packet wise transmission. The second difference is on the device identification. Specifically, in order for the $\mathrm{HG}$ to communicate over a Bluetooth connection, the HG has to bind a port e.g. / rfcomm0 to a specific device. That distinction effectively uniquely defines the receiver through the specific port. This is not the case in IEEE 802.15.4 based communication due to the connectionless approach followed. In this case the $\mathrm{HG}$ communicates through a USB port only with a mote, effectively acting as a proxy for the whole 802.15.4 WSN as indicated in Fig. 3.

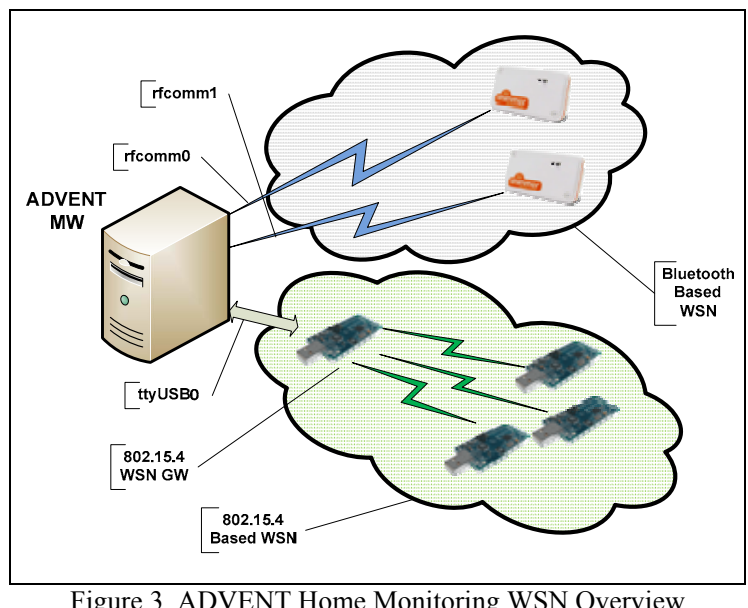

Figure 3. ADVENT Home Monitoring WSN Overview

Consequently, substantial modifications were required so that HG could uniquely distinguish each 802.15.4 mote. To achieve this goal, the receiver of a packet transmitted by 
the HG is defined on the packet itself (through a respective packet field) and then utilized by the IEEE 802.15.4 communicating motes exploiting the capability of a unicast packet transmission instead of multicast. The application running at the mote acting as an 802.15.4 gateway resolves the destination address of each packet and integrates respective information to the header of each transmitted packet. This entails the overall packet format awareness by the 802.15.4 gateway's firmware but reduces the complexity of application layer of every other 802.15.4 mote.

Aiming towards code reusability as a primary concern the implementation of the WSN motes' application logic is agnostic to the underlying communication technology. To achieve this characteristic, since the implementation is based on TinyOS [13], a new nesC component was created that wraps the already existing communication component, thus exposing the same interface towards the application for both Bluetooth and IEE 802.15.4 case. It is noted that we did not use a predefined payload format and thus we allow packets of different sizes to be transmitted without considering different manipulation. By this decision we aim at enhancing extensibility and versatility of our application.

\section{Low level HG - 802.15.4 WSN Interface}

Significant effort is also put on low level data exchange interface between HG and the IEEE 802.15.4 WSN gateway mote as depicted in Fig. 3. The original host application supporting only Bluetooth communication assumed that a port is already bind to a Bluetooth device and thus communication was straightforward. In order to send packets to the motes via 802.15 .4 we used the mechanism provided by the TinyOS SDK and implemented an efficient Python based program enabling bi-directional communication between the ADVENT HG and the mote attached to the USB port (effectively the gateway towards the IEEE 802.15.4 WSN). When activated the implemented Python program opens a server side socket encapsulating data coming from the $\mathrm{HG}$ to predefined 802.15.4 packets and sends it via serial to 802.15 .4 gateway and then to the IEEE 802.15.4 part of the home environment. On the other hand, it uses objects of classes provided by the TinyOS Python SDK, in order to read respective packets from the serial. When a packet arrives, our receive method reads its payload and writes its content to our socket. From the HG side, a new class to act as a medium between the application layer and the 802.15.4 gateway was required. Specifically, the respective class implements a serial port interface effectively making IEEE 802.15.4 transparent to the HG application. Upon creation it opens a socket, listening to a predetermined port. When a serial port is selected, the previously analyzed python script is executed. Finally, it establishes a connection to the socket opened by the python script.

\section{CONCLUSIONS}

As it is projected personalized and versatile home monitoring will attract active interest and even comprise a necessity in the next years. In that respect appropriate platforms will be required to seamlessly interconnect sensors using different and diverse communication technologies, devices offering various types of sensors of different capabilities. Even more, such platforms will be utilized by user characterized by different requirements, needs, conditions and background knowledge. Currently respective available platforms exhibit a significant degree of incompatibility prohibiting the implementation of a truly comprehensive and flexible home monitoring environment. In this context this paper presents a detailed design and implementation effort undertaken to offer a novel home monitoring environment that provides a high degree of heterogeneity support in various aspects. Specifically addressed implementation challenges concerning both the WSN motes and the HG software architectures are presented and analyzed based on extending existing components. Proposed design and implementation approaches so as to achieve communication technology agnostic applications are also presented. Furthermore, design and implementation extensions so as to support different vendors and various types of sensor also comprise a significant feature of the targeted platform. As future work and envisioning a continuously evolving complete end-to-end solution effort will be devoted in further extending heterogeneous capabilities of the platforms. Additionally a critical direction concerns extending respective $\mathrm{HG}$ functionality towards backhaul communication towards 3rd part service providers and remote monitoring services.

\section{ACKNOWLEDGEMENT}

The research described in this paper has been funded by the Project

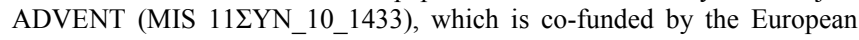
Regional Development Funds and the National Fund in the context of Programme Cooperation II-2011

\section{REFERENCES}

[1] European Commision, "The 2012 Ageing Report. Economic and budgetary projections for the 27 EU Member States (2010-2060)", European Economy, 2|2012

[2] Panagiotakopoulos, et.al.. "Exploring Ambient Assisted Living Job Profiles", 6th PETRA, ACM, 2013, Rhodes, Greece

[3] Centers for Disease Control and Prevention. Chronic Disease Overview: Costs of Chronic Disease. Centers for Disease Control and Prevention Web site. Available at http://www.cdc.gov/nccdphp/overview.htm. Accessed June 23, 2014.

[4] Holmes, AM. Deb, P., "The effect of chronic illness on the psychological health of family members.", J. Ment. Health Policy Econ., Vol 6, No 1, pp. 13-22, 2003.

[5] van den Broek, G., Cavallo, F., and Wehrmann, C. (2010). AALIANCE Ambient Assisted Living Roadmap. Ambient Intelligence and Smart Environments, IOS Press

[6] Alemdar, H., Ersoy, C.: Wireless sensor networks for healthcare: A survey. Elsevier Computer Networks 54, 2688-2710 (2010)

[7] Lecointre, A., et. al.: Miniaturized Wireless Sensor Networks. In: International Semiconductor Conference, pp. 13-17, Sinaia (2006)

[8] Jin, M.-H.et. al.: Sensor Network Design and Implementation for Health Telecare and Diagnosis Assistance Applications. In: 11th ICPADS, Fukuoka (2005)

[9] Pantelopoulos, A et. al. "A Survey on Wearable Sensor-Based Systems for Health Monitoring and Prognosis" IEEE Transactions on Systems, Man, and Cybernetics(Volume:40, Issue: 1 )

[10] Butun, I, "A Survey of Intrusion Detection Systems in Wireless Sensor Networks" IEEE Communications Surveys \& Tutorials (Volume:16, Issue: 1)

[11] WSN_TelosB_Platform, http://www.willow.co.uk/TelosB_Datasheet.pdf

[12] WSN Shimmer Platform, http://www.shimmersensing.com/

[13] TinyOS WSN Operating System: http://www.tinyos.net/

[14] IEEE 802.15 (TG4). http://www.ieee802.org/15/pub/TG4.html

[15] Bluetooth. (2009, Jun. 15). [Online]. Available: www.bluetooth.com

[16] GNU/Linux: https://www.gnu.org/gnu/linux-and-gnu.html

[17] Python: https://www.python.org/

[18] PyBluez: https://code.google.com/p/pybluez/

[19] Th. Panagiotakopoulos, et. al, "Taking Care of Elderly People with Chronic Conditions using Ambient Assisted Living technology: The ADVENT perspective" HCI International, 22-27/6/2014, Crete, Greece 